\title{
TIPO ACROMIAL DE BRASILEIROS: ESTUDO EM ESCÁPULAS HUMANAS
}

\section{ARTIGO ORIGINAL}

CARDINOT, Themis Moura ${ }^{1}$

MEDIANO, Mauro Felippe Felix ${ }^{2}$

ALMEIDA, Jamille Santos de ${ }^{3}$

${ }^{1}$ Professora Associada de Anatomia Humana na Universidade Federal Rural do Rio de Janeiro (UFRRJ) (2010-atual). Pesquisadora na Disciplina de Ortopedia da Faculdade de Ciências Médicas da Universidade do Estado do Rio de Janeiro (UERJ) (2010-atual). Docente de Anatomia Humana da ABEU Centro Universitário de Belford Roxo/RJ (2007-2010). Graduação em Letras (UFF/2003). Graduação em Licenciatura Plena em Educação Física (UERJ/2004). Doutorado em Ciências Médicas (FMUSP/2009).

2 Pesquisador no Instituto Nacional de Infectologia Evandro Chagas da Fundação Oswaldo Cruz/RJ (INI/FIOCRUZ) e no Instituto Nacional de Cardiologia - Fundação Pro-Coração (INC/FUNDACOR). Graduação em Licenciatura Plena em Educação Física (UERJ/2004). Mestrado em Nutrição Humana (UFRJ/2006). Doutorado em Fisiopatologia Clínica e Experimental (UERJ/2010). Pós-doutorado em Saúde Coletiva/Epidemiologia (UERJ/2012). Graduação em Fisioterapia (UNESA/2016).

3 Professora de Educação Física na Academia Velox Fitness. Graduação em Licenciatura Plena em Educação Física (UERJ/2008). Especialização em Fisiologia do Exercício - Avaliação Morfofuncional (Universidade Gama Filho/2012). Bacharelado em Fisioterapia (IBMR/2019). 
COSTA, Bruno Ferreira Alves Castello da ${ }^{4}$

AZEVEDO, Paula de Abreu ${ }^{5}$

BRITO, Rodrigo Paiva Martins de ${ }^{6}$

BORGES, Juliana Pereira ${ }^{7}$

VASCONCELLOS, Fabrício Vieira do Amaral ${ }^{8}$

${ }^{4}$ Technical Advisor na empresa Rio Surf Survive (2018-atual). Technical Advisor na empresa SurfingPedagogy.com (2005-atual). Graduação em Licenciatura Plena em Educação Física (UERJ/2005). Graduação em Engenharia de Produção (UFRJ/2003). 5 Professora Sócia da MEX - Medicina do Exercício (2011-atual). Graduação em Licenciatura Plena em Educação Física (UERJ/2005).

6 Pesquisador do Instituto Brasil de Tecnologia da Saúde (IBTS/2019-atual). Graduação em Licenciatura Plena em Educação Física (UERJ/2006). Especialização em Biomecânica (UFRJ/2010). Graduação em Bacharelado em Fisioterapia (Universidade Estácio de Sá/2011). Especialização em Osteopatia (Escuela de Osteopatia de Madrid/2015).

7 Professora Adjunta na Universidade do Estado do Rio de Janeiro e Docente Permanente do Programa de Pós-Graduação em Ciências do Exercício e do Esporte (PPGCEE/UERJ). Jovem Cientista do Nosso Estado pela FAPERJ. Pesquisadora do Laboratório de Atividade Física e Promoção da Saúde (LABSAU), onde coordena o projeto de extensão Vida Positivo: Exercício Físico Para Pessoas Vivendo com HIV/AIDS. Graduação em Licenciatura Plena em Educação Física (UERJ/2004). Especialização em Reabilitação Cardíaca (INCOR-USP/2007). Mestrado em Ciências Médicas (UERJ/2010). Doutorado em Biologia Celular e Molecular (FIOCRUZ/2014). ${ }^{8}$ Professor Adjunto da disciplina de Futebol de Campo no Instituto de Educação Física e Desportos da Universidade do Estado do Rio de Janeiro. Coordenador do Laboratório de Estudos em Futebol (LABESFUT) da Universidade do Estado do Rio de Janeiro e Docente Permanente do Programa de Pós-Graduação em Ciências do 
RÉGIS, Márcio Trindade Duque Estrada ${ }^{9}$

SILVA, Michelle Gomes Moreira da ${ }^{10}$

VERDOORN, Karine Silva ${ }^{11}$

LUCAS, Isabela Henriques ${ }^{12}$

Exercício e do Esporte (PPGCEE/UERJ). Graduação em Licenciatura Plena em Educação Física (UERJ/2005). Pós-graduação em Treinamento Desportivo (Universidade Gama Filho/2007). Mestrado em Ciência da Motricidade Humana (Universidade Castelo Branco/2009). Doutorado em Ciências do Desporto (Universidade do Porto-Portugal/2014).

${ }^{9}$ Graduação em Licenciatura Plena em Educação Física (UERJ/2006). Mestrado em Educação em Ciências e Saúde (UFRJ/2009). Especialização em Ergonomia (COPPE-UFRJ/2011).

10 Professora de Educação Física na Prefeitura da Cidade do Rio de Janeiro. Graduação em Licenciatura Plena em Educação Física (UERJ/2005). Graduação em Pedagogia (UERJ/CEDERJ). Especialização em Pedagogia Empresarial (Instituto a Vez do Mestre/UCAM).

11 Professora Adjunta de Fisiologia Humana na Universidade Federal do Rio de Janeiro (UFRJ - Campus Macaé/RJ). Graduação em Licenciatura Plena em Educação Física (UERJ/2005). Mestrado em Ciências Biológicas/Biofísica (UFRJ/2008). Doutorado em Ciências Biológicas/Fisiologia (UFRJ/2013). Pós-Doutorado (UFRJ/2014).

12 Professora de Educação Física na Rede Sarah de Hospitais de Reabilitação. Graduação em Licenciatura Plena em Educação Física (UERJ/2005). Graduação em Fisioterapia (Centro Universitário Metodista Bennett/2009). Mestrado (2012) e Doutorado (2015) em Ciências Biológicas (Fisiologia) no Instituto de Biofísica Carlos Chagas Filho da Universidade Federal do Rio de Janeiro. 
CARVALHO, Juliana Rangel de Oliveira ${ }^{13}$

MONIZ-DE-ARAGÃO, Afonso Henriques Bandeira de ${ }^{14}$

CARDINOT, Themis Moura. Et al. Tipo acromial de brasileiros: Estudo em escápulas humanas. Revista Científica Multidisciplinar Núcleo do Conhecimento. Ano 05, Ed. 03, Vol. 06, pp. 17-28. Março de 2020. ISSN: 2448-0959, Link de acesso: https://www.nucleodoconhecimento.com.br/saude/tipo-acromial-debrasileiros

\section{RESUMO}

O estudo do acrômio tem sido motivo de interesse devido ao seu papel na patogênese, diagnóstico e tratamento conservador ou cirúrgico da dor no ombro. A maioria dos estudos que avaliou o tipo acromial foi realizada na América do Norte, Europa e Ásia e, por isso, pouco se sabe sobre o perfil acromial em brasileiros. O objetivo desse estudo foi avaliar o tipo acromial de brasileiros. Um total de 112 escápulas secas, em bom estado de conservação, foram analisadas para esse estudo. As escápulas de

13 Professora Preceptora de MorfoMédica e MorfoFisiologia na Universidade do Grande Rio (UNIGRANRIO) para os cursos de Medicina, Odontologia, Fisioterapia, Enfermagem, Farmácia, Radiologia, Educação Física e Nutrição. Graduação em Educação Física (ABEU Centro Universitário/2010). Especialização em Anatomia Humana (IBMR/2012). Foi bolsista discente do projeto de pesquisa "Dor no ombro: análise radiográfica do morfotipo acromial de Brasileiros", pela ABEU Centro Universitário de Belford Roxo/RJ, sob orientação da professora Themis Moura Cardinot.

14 Professor da disciplina de Anatomia Humana para os cursos de Medicina e Educação Física na Universidade do Estado do Rio de Janeiro (UERJ). Coordenador da disciplina de Anatomia Humana para o curso de Educação Física na UERJ. Graduação em Medicina (UERJ/1989). Mestrado em Morfologia (UERJ/2000). Especialista e membro titular das Sociedades Brasileiras de Coloproctologia e de Endoscopia Digestiva. 
ambos os lados foram avaliadas por dois observadores que utilizaram a classificação de Bigliani (tipo I - plano, tipo II - curvo, tipo III - gancho) refinada por Epstein. Os dados coletados por cada observador foram registrados em planilhas individuais e um consenso sobre o tipo acromial foi estabelecido para cada um dos acrômios avaliados. Das 112 escápulas analisadas foram encontradas 54 (48,2\%) do tipo I (plano), 53 $(47,3 \%)$ do tipo II (curvo) e 5 (4,5\%) do tipo III (gancho). A análise de 112 escápulas humanas secas mostrou que o tipo acromial de maior incidência em brasileiros são os tipos I e II. O tipo III apresentou uma ocorrência muito pequena na amostra estudada.

Palavras-chave: Acrômio, ombro, dor, espaço subacromial, síndrome do impacto.

\section{INTRODUÇÃO}

A dor no ombro é uma queixa comum no consultório do ortopedista e o tipo acromial tem sido implicado em sua gênese (YADAV; ZHU, 2017). O acrômio é uma proeminência óssea da escápula, que se curva lateral e anteriormente sobre a articulação do ombro, e se articula com a clavícula, formando a articulação acromioclavicular (GRAY, 1985). (Figura 1)

Figura 1: Articulação do ombro e seus elementos ósseos.
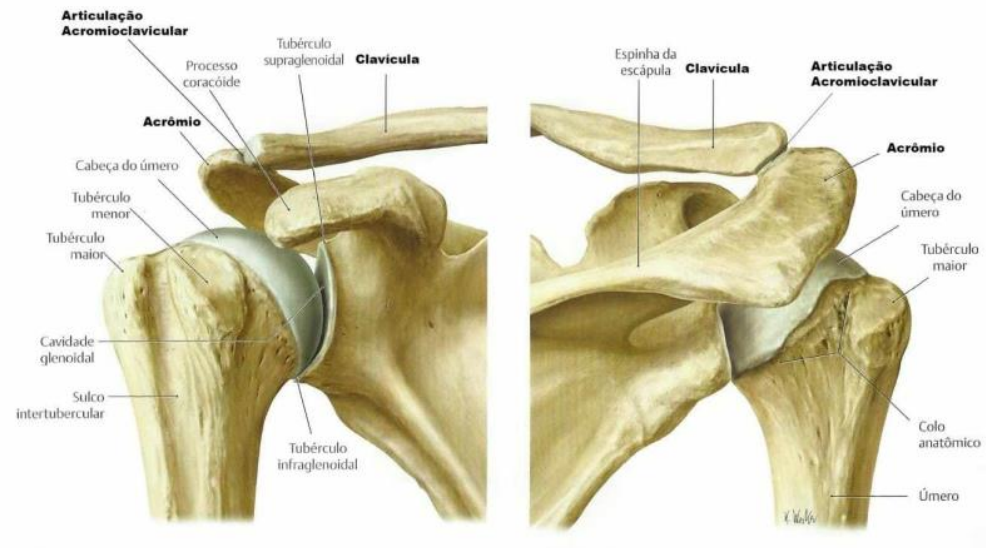

Fonte: GILROY; MACPHERSON; ROSS, 2011. 
Hamilton (1875) foi o primeiro autor a identificar o tipo acromial como uma potencial fonte de sintomas no ombro. Dentre as diversas causas do ombro doloroso, as lesões das estruturas periarticulares (bursa subacromial e tendões do manguito rotador), localizadas no espaço subacromial (região entre o arco coracoacromial e o tubérculo maior do úmero) são as mais comuns (MICHENER; MCCLURE; KARDUNA, 2003; YADAV; ZHU, 2017). (Figura 2)

Figura 2: Articulação do ombro e suas estruturas periarticulares.

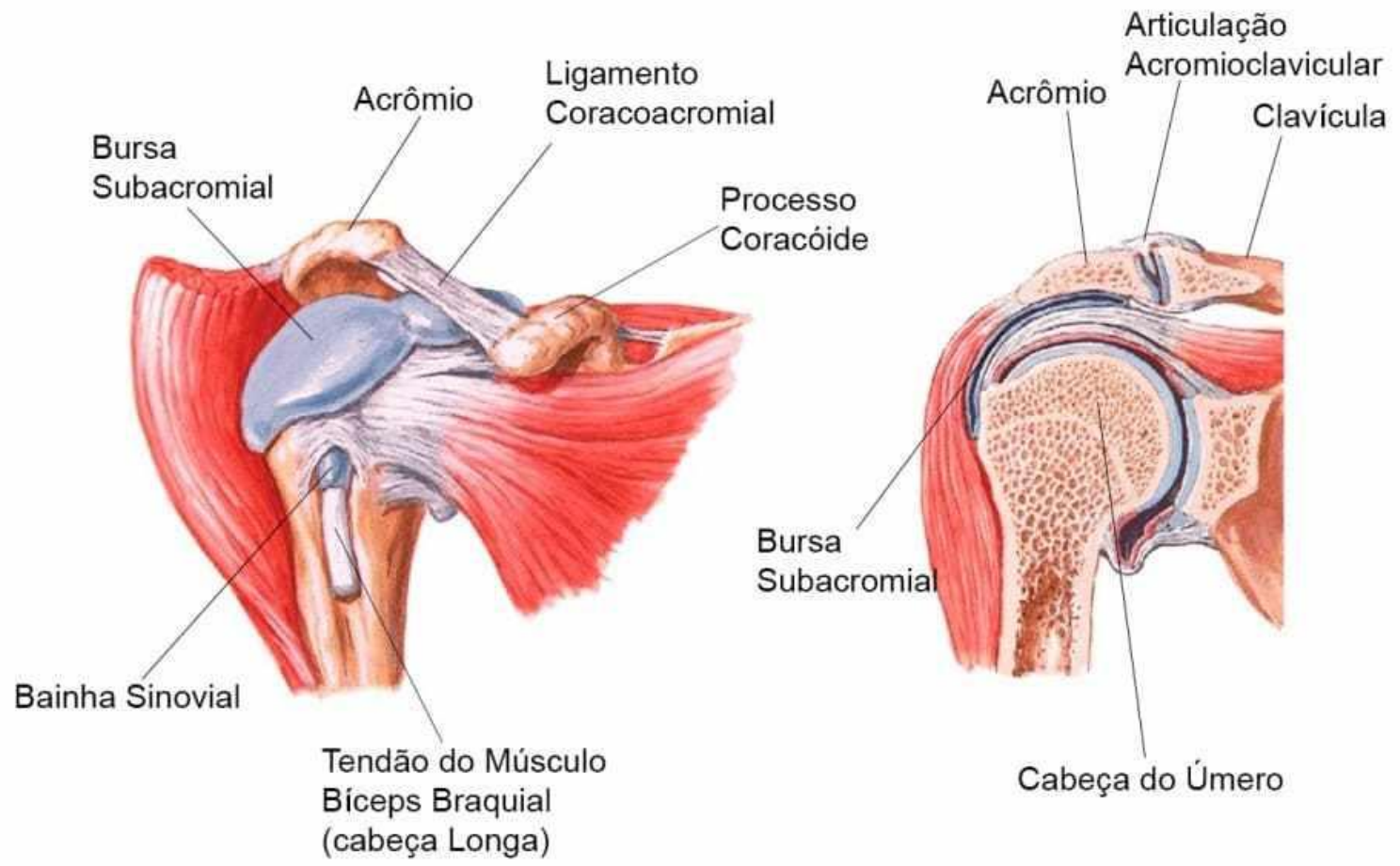

Fonte: NETTER, 2004.

Bigliani et al. (1986) classificaram o acrômio em três tipos, de acordo com o formato de sua superfície inferior: tipo I (plano), tipo II (curvo) e tipo III (gancho). (Figura 3) 
Figura 3: Desenho esquemático da classificação de Bigliani para tipo acromial (tipo I - plano, tipo II - curvo, tipo III - gancho) refinada por Epstein (tipo II: declive no terço médio; tipo III: declive no terço anterior).
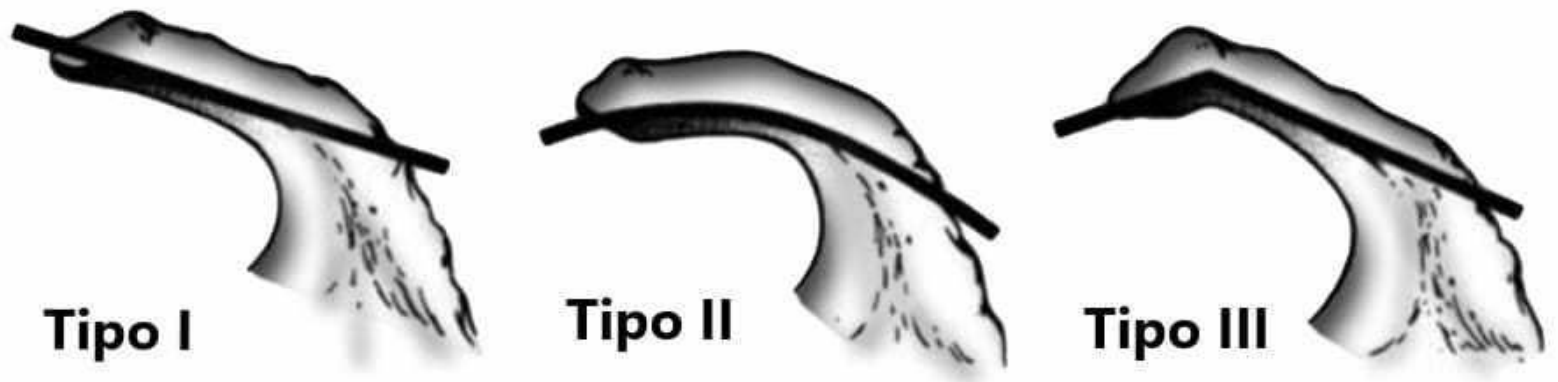

Fonte: Epstein et al., 1993.

Os tipos acromiais II e III (curvo e gancho), por causa do seu formato, implicam em um estreitamento do espaço subacromial (Figura 4), levando ao atrito e impacto excessivos e sucessivos das estruturas periarticulares contra o próprio acrômio (MORRISON; BIGLIANI, 1987; EPSTEIN et al, 1993; BIGLIANI; LEVINE, 1997).

Figura 4: Desenho esquemático do estreitamento do espaço subacromial devido ao tipo acromial.

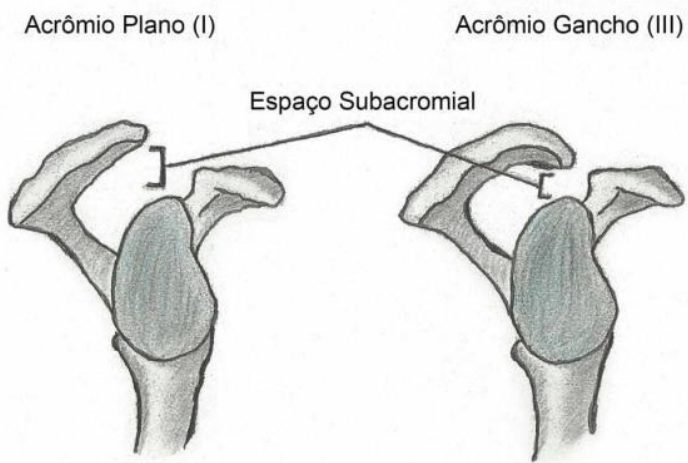

Fonte: Os autores. 
Esse processo desempenha papel fundamental na instalação da síndrome do impacto e no mecanismo das doenças do manguito rotador (EPSTEIN et al, 1993; YADAV; ZHU, 2017). (Figura 5)

Figura 5: llustração do mecanismo da síndrome do impacto no ombro e suas estruturas lesionadas.
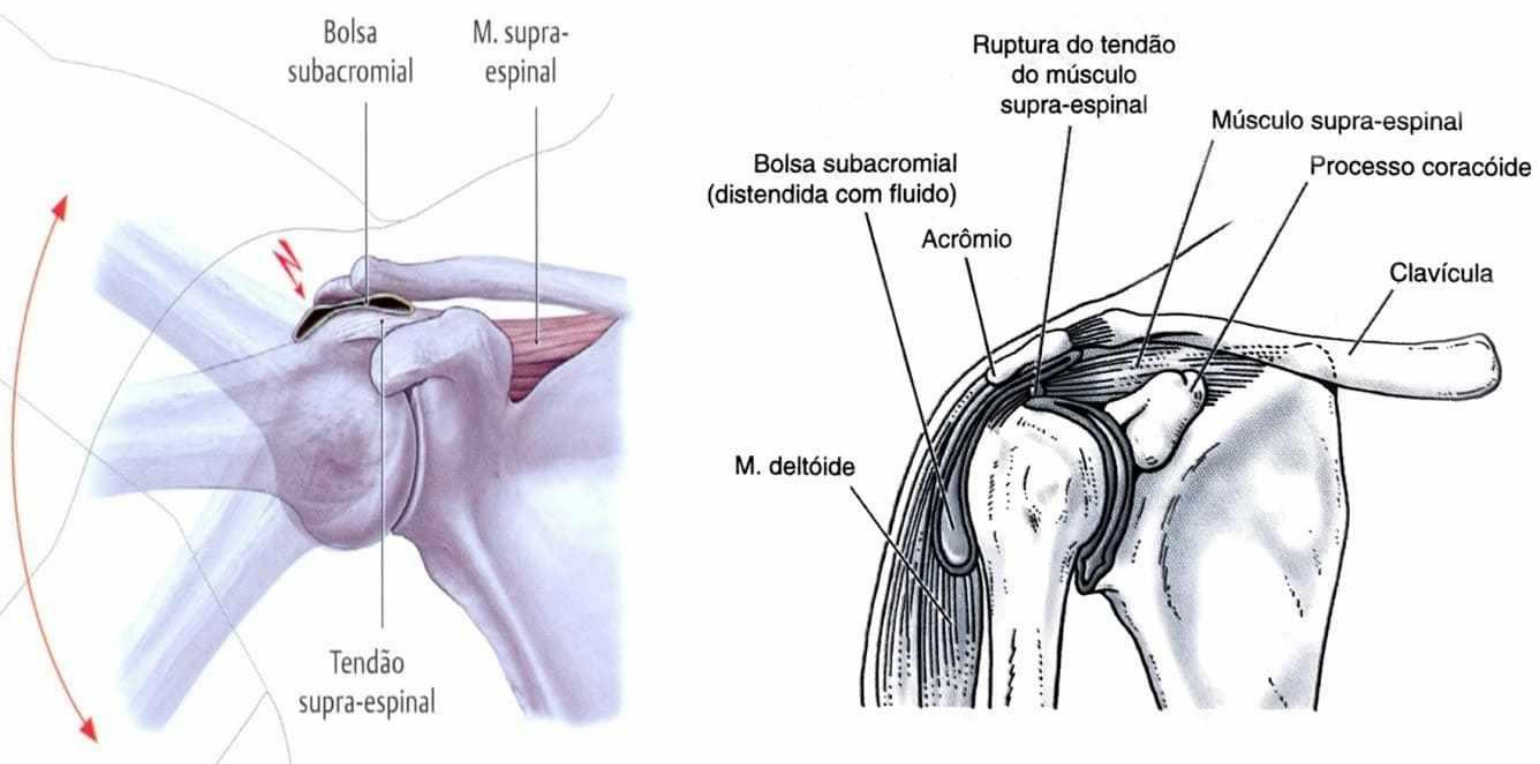

Fonte: MOORE; AGUR, 2004; SCHÜNKE; SCHULTE; SCHUMACHER, 2006.

O estudo do acrômio tem sido motivo de interesse devido ao seu papel na patogênese, diagnóstico e tratamento conservador ou cirúrgico da dor no ombro. A maioria dos estudos que avaliou o tipo acromial foi realizada na América do Norte, Europa e Ásia e, por isso, pouco se sabe sobre a incidência dos tipos acromiais, segundo a classificação de Bigliani et al. (1986), em brasileiros. Dessa forma, o objetivo desse estudo foi avaliar o perfil do tipo acromial de brasileiros, em escápulas humanas.

\section{MATERIAIS E MÉTODOS}

Um total de 112 escápulas humanas secas, em bom estado de conservação, foram analisadas para esse estudo, no ano de 2002. As escápulas pertenciam às coleções da Universidade do Estado do Rio de Janeiro, Universidade Federal do Rio de Janeiro, 
Universidade Estácio de Sá/RJ, Faculdade de Medicina de Petrópolis/RJ e da Escola Paulista de Medicina/SP. Todas as escápulas pertenciam a espécimes adultos, de gênero e idade desconhecidos.

As escápulas, de ambos os lados, foram avaliadas e classificadas por dois observadores que utilizaram a classificação de Bigliani et al. (1986) (tipo I - plano, tipo II - curvo, tipo III - gancho) refinada por Epstein et al. (1993). Este autor propôs para o tipo II a presença de um declive em seu terço médio, e o tipo III, apresentaria tal declive em seu terço anterior. (Figura 3)

Os dados coletados por cada observador foram registrados em planilhas individuais e um consenso sobre o tipo acromial foi estabelecido para cada um dos acrômios avaliados. A análise estatística foi realizada usando-se variáveis representadas descritivamente em tabelas de contingência contendo frequências absolutas (n) e relativas (\%) e a associação entre elas foi avaliada com o teste de Fisher.

\section{RESULTADOS}

Foram encontradas frequências distintas para a ocorrência dos diferentes tipos acromiais. Das 112 escápulas analisadas foram encontradas 54 (48,2\%) do tipo I (plano), 53 (47,3\%) do tipo II (curvo) e 5 (4,5\%) do tipo III (gancho). (Tabela 1)

Tabela 1: Ocorrência dos tipos acromiais em escápulas secas de brasileiros

\begin{tabular}{|l|l|l|}
\hline Tipo acromial & n & $\%$ \\
\hline I & 54 & 48,2 \\
\hline II & 53 & 47,3 \\
\hline III & 5 & 4,5 \\
\hline Total & $\mathbf{1 1 2}$ & $\mathbf{1 0 0}$ \\
\hline
\end{tabular}

Legenda: frequências absolutas $(n)$ e relativas (\%)

A Figura 6 ilustra alguns exemplares dos tipos acromiais I (plano), II (curvo) e III (gancho) encontrados na amostra de escápulas secas estudadas. 
Figura 6: Fotografia de alguns exemplares dos tipos acromiais I, II e III nas escápulas estudadas.

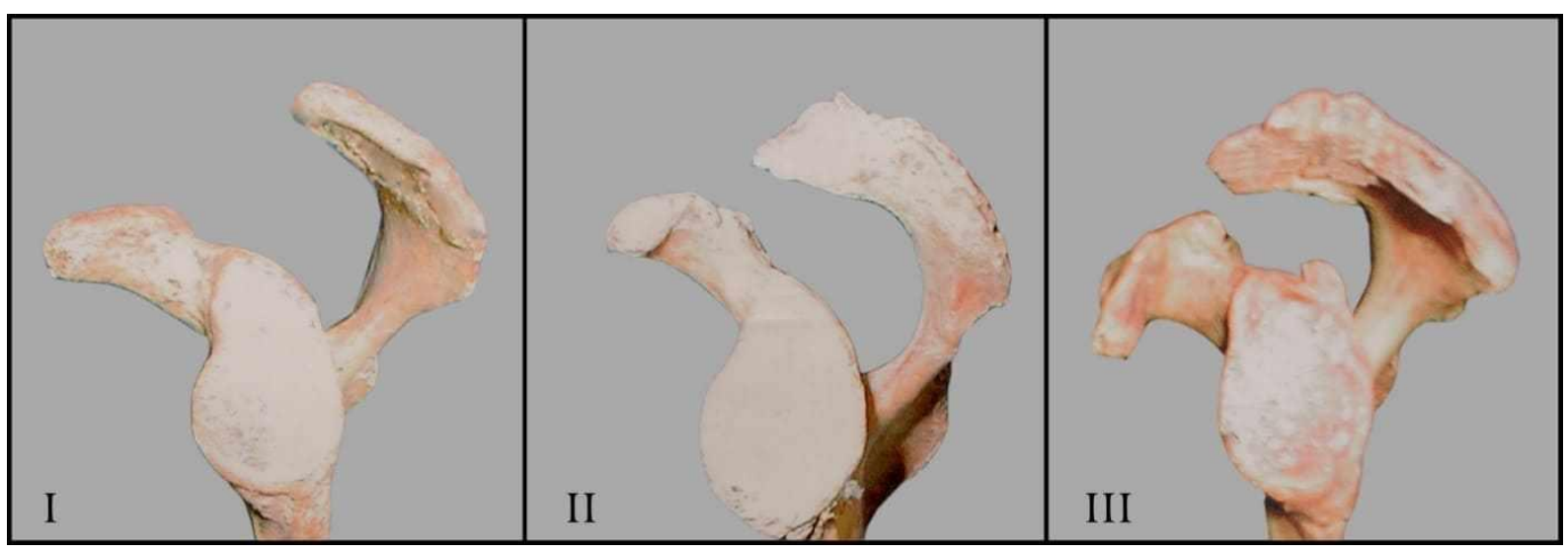

Fonte: Os autores.

\section{DISCUSSÃO}

A classificação do tipo acromial surgiu a partir do estudo de Bigliani et al. (1986) que, utilizando escápulas secas e considerando a superfície inferior do acrômio, classificaram-no em três tipos: tipo I (plano), tipo II (curvo) e tipo III (gancho). Nesse estudo não foram apresentadas fotografias ou diagramas que indicassem os limites pretendidos para cada categoria, e a interpretação foi guiada apenas pelos significados cotidianos das palavras plano, curvo e gancho. Epstein et al. (1993) resolveram essa dificuldade propondo que, se a angulação do acrômio ocorresse em seu terço médio, este deveria ser considerado curvo, e se a angulação ocorresse no terço anterior, o acrômio deveria ser considerado gancho.

Vários estudos analisando a incidência do tipo acromial foram realizados em países europeus. Coskun et al. (2006) estudaram 90 escápulas secas, de idade e sexo desconhecidos; e 90 pacientes adultos, em uma população turca. Paraskevas et al. (2008) estudaram 44 pares de escápulas secas, em uma população grega. Natsis et al. (2007) examinaram 423 escápulas secas, de idade e sexo desconhecidos, em uma amostra alemã. Os autores utilizaram o método de Bigliani et al. (1986) para a classificação do tipo acromial. Os três estudos apresentaram predominância do tipo acromial II. 
Diversos estudos foram desenvolvidos na Ásia sobre o perfil do tipo acromial. Sangiampong et al. (2007) examinaram 154 escápulas tailandesas secas. Jacinth et al. (2018) e Gosavi et al. (2015) estudaram 40 e 127 escápulas secas, de idade e sexo desconhecidos, em uma população indiana, respectivamente. Guo et al. (2018) examinaram 292 escápulas secas em uma população chinesa. Todos esses autores classificaram o tipo acromial de acordo com Bigliani et al. (1986). Esses estudos mostraram que o acrômio tipo II foi o mais predominante no continente asiático.

Alguns poucos estudos sobre o acrômio foram realizados na América do Sul. Collipal et al. (2010) estudaram 36 escápulas de idade e sexo desconhecidos, em uma população chilena. Aragão et al. (2014) estudaram 90 escápulas secas, de idade e sexo desconhecidos, em uma população brasileira. Ambos os estudos classificaram os acrômios de acordo com Bligliani et al. (1986) e concluíram que o acrômio tipo II foi o mais predominante.

Nosso estudo, feito em escápulas humanas secas, mostrou uma incidência equivalente entre os acrômios tipo I $(48,2 \%)$ e tipo II $(47,3 \%)$. Acreditamos que a classificação dos tipos acromiais feita de forma subjetiva, como é o método de Bigliani et al. (1986), tenha limites muito sutis entre cada tipo acromial, porque o senso de interpretação varia para cada observador. Aliado ao fato de que nosso estudo foi realizado por vários observadores em diferentes momentos, o ideal seria que um número menor e que os mesmos observadores realizassem a avaliação do tipo acromial para diminuir o viés do método.

\section{CONSIDERAÇÕES FINAIS}

A análise de 112 escápulas humanas secas mostrou que o tipo acromial de maior incidência em brasileiros são os tipos I e II. O tipo III apresentou uma ocorrência muito pequena na amostra estudada. Mais estudos são necessários, preferencialmente em que gênero e idade sejam conhecidos, para se traçar o perfil do tipo acromial da população brasileira. 


\section{REFERÊNCIAS}

ARAGÃO, J.A.; SILVA, L.P.; REIS, F.P.; MENEZES, C.S.S. Analysis on the acromial curvature and its relationships with the subacromial space and types of acromion. Revista Brasileira de Ortopedia, v. 49, n. 6, p. 636-41, 2014.

BIGLIANI, L.U.; LEVINE, W.N. Current concepts review - Subacromial impingement syndrome. Journal of Bone and Joint Surgery - American, v. 79, p. 1854-68, 1997.

BIGLIANI, L.U.; MORRISON, D.S.; APRIL, E.W. The morphology of the acromion and rotator cuff impingement (Abstr). Journal of Orthopaedic Translation, v.10, p. 228, 1986.

COLLIPAL, E.; SILVA, H.; ORTEGA, L.; ESPINOZA, E.; MARTÍNEZ, C. The acromion and its different forms. International Journal of Morphology, v. 28, n. 4, p. 1189-92, 2010.

COSKUN, N.; KARAALI, K.; CEVIKOL, C.; DEMIREL, B.M.; SINDEL, M. Anatomical basics and variations of the scapula in Turkish adults. Saudi Medical Journal, v. 27, n. 9 , p. 1320-5, 2006.

EPSTEIN, R.E.; SCHWEITZER, M.E.; FRIEMAN, B.G.; FENLIN, JR.; JOHN, M.; MITCHELL, D.G. Hooked acromion: Prevalence on MR images of painful shoulders. Radiology, v. 187, p. 479-81, 1993.

GILROY, A.M.; MACPHERSON, B.R.; ROSS, L.M; baseado no trabalho de SCHUENKE, M.; SCHULTE, E.; SCHUMACHER, U. Atlas de Anatomia. Rio de Janeiro: Guanabara Koogan, 2011.

GOSAVI, S.; JADHAV, S.; GARUD, R. Morphometry of acromion process: a study of Indian scapula. International Journal of Pharma Research and Health Sciences, v. 3, n. 5, p. 831-5, 2015.

GRAY, H. Anatomy of the Human Body. Philadelphia: Lea \& Febiger, 1985. 
GUO, X.; OU, M.; YI, G.; QIN, B.; WANG, G.; FU, S.; ZHANG, L. Correction between the morphology of acromion and acromial angle in Chinese population: a study on 292 scapulas. BioMed Research International, v. 2018, p. 1-6, 2018.

HAMILTON, F.H. Fracture of the scapulas. A practical treatise on fractures and dislocations. 5 ed. Philadelphia: Henry C. Lea, p. 209-21, 1875.

JACINTH, J.S.; PUSHPAM, E.S.; SLATER, S.; MUNIYAPPAN, V. Morphometry of the acromion process of human scapulae and its clinical implications. Journal of Evolution of Medical and Dental Sciences, v. 7, n. 18, p. 2205-9, 2018.

MICHENER, L.A.; McCLURE, P.W.; KARDUNA, A.R. Anatomical and biomechanical mechanisms of subacromial impingement syndrome. Clinical Biomechanics, v. 18, p. 369-79, 2003.

MOORE, K.L.; AGUR, A.M.R. Fundamentos de Anatomia Clínica. 2 ed. Rio de Janeiro: Guanabara Koogan, 2004.

MORRISON, D.S.; BIGLIANI, L.U. The clinical significance of variation in acromial morphology (abstr). Journal of Orthopaedic Translation, v. 11, p. 234, 1987.

NATSIS, K.; TSIKARAS, P.; TOTLIS, T.; GIGIS, I.; SKANDALAKIS, P.; APPELL, H.J.; KOEBKE, J. Correlation between the four types of acromion and the existence of enthesophytes: a study on 423 dried scapulas and review of the literature. Clinical Anatomy, v. 20, p. 267-72, 2007.

NETTER, F.H. Atlas de Anatomia Humana. 3 ed. Porto Alegre: Artmed, 2004.

PARASKEVAS, G.; TZAVEAS, A.; PAPAZIOGAS, B.; KITSOULIS, P.; NATSIS, K.; SPANIDOU, S. Morphological parameters of the acromion. Folia Morphologica, v. 67 , n. 4, p. 255-60, 2008.

SANGIAMPONG, A.; CHOMPOOPONG, S.; SANGVICHIEN, S.; THONGTONG, P.; WONGJITTRAPORN, S. The acromial morphology of Thais in relation to gender and 
age: study in scapular dried bone. Journal of the Medical Association of Thailand, v. 90 , n. 3, p. 502-7, 2007.

SCHÜNKE, M.; SCHULTE, E.; SCHUMACHER, U. Prometheus: Atlas de Anatomia. Rio de Janeiro: Guanabara Koogan, 2006.

YADAV, S.K.; ZHU, W.H. A systematic review: Of acromion types and its effect on degenerative rotator cuff tear. International Journal of Orthopaedics Sciences, v. 3 , n. 1, p. 453-8, 2017.

\section{NOTA DOS AUTORES}

Esse estudo foi desenvolvido por um grupo de monitores de anatomia humana da Universidade do Estado do Rio de Janeiro, que não se limitaram apenas a realizar suas atividades didático-acadêmicas, mas se dedicaram também à pesquisa científica de temas relacionados ao estudo da anatomia humana macroscópica e suas implicações clínicas, sob a orientação e supervisão do professor Afonso Henriques Bandeira Moniz-de-Aragão (Figura 7).

Figura 7: Professor Afonso Moniz-de-Aragão e os monitores de Anatomia Humana para o curso de Educação Física durante os anos de 2002 a 2003.

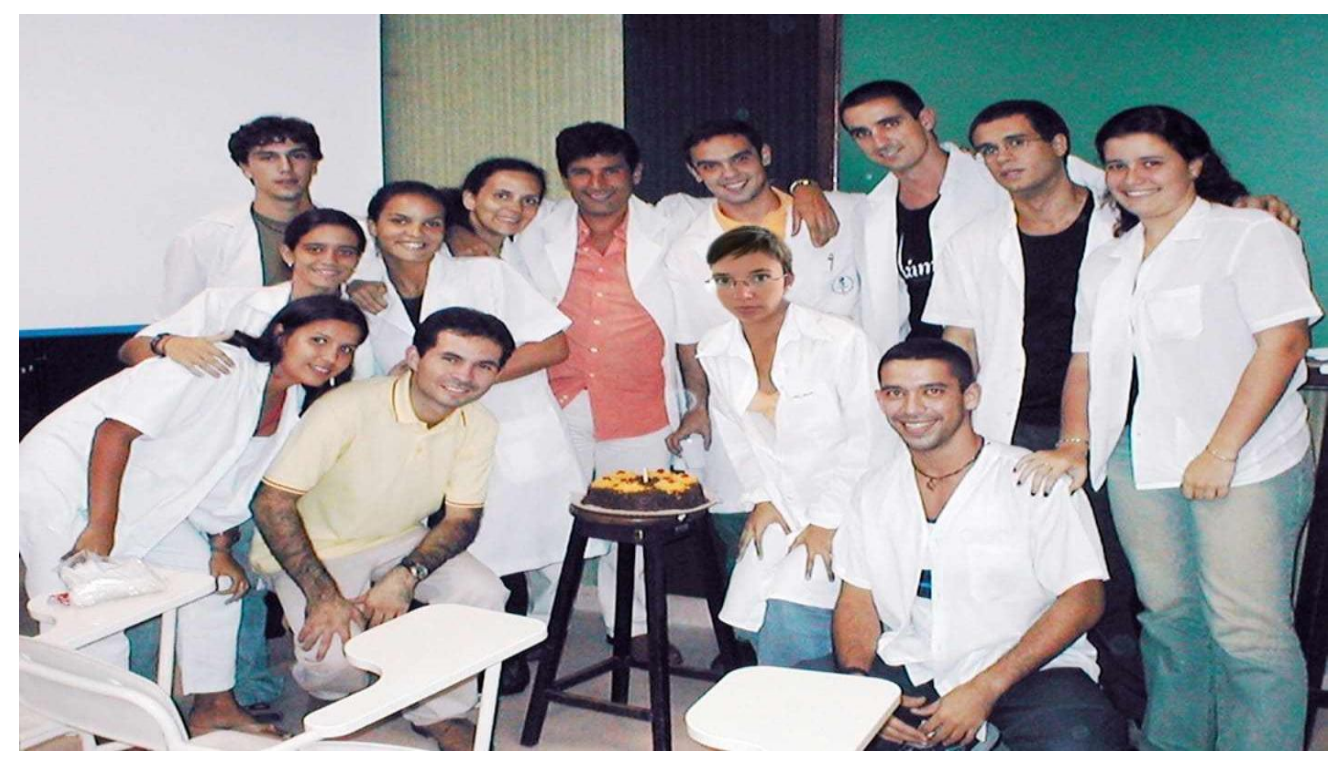

Fonte: Os autores. 
Enviado: Novembro, 2019.

Aprovado: Março, 2020. 